\title{
Hybrid Tamm Plasmon \\ Photonic Quasicrystal Cavity with an Organic Active Region
}

\author{
K.M. Morozov ${ }^{a, b, *}$, A.V. BelonovskiI ${ }^{a}$, K.A. IVAnOV $^{b}$, E.I. Girshova ${ }^{a}$ \\ AND M.A. KALITEEVSKI ${ }^{a, b, c}$ \\ ${ }^{a}$ St. Petersburg Academic University, Khlopina 8/3, 194021 St. Petersburg, Russia \\ ${ }^{b}$ ITMO University, Kronverkskiy pr. 49, 197101 St. Petersburg, Russia \\ ${ }^{c}$ Ioffe Institute, Politekhnicheskaya 26, 194021 St. Petersburg, Russia
}

\begin{abstract}
We theoretically investigate a hybrid Tamm plasmon cavity with a quasicrystal symmetry structure. The latter is replacing the usual distributed Bragg reflector with an organic (4,4'-bis(N-carbazolyl)-1,1'-biphenyl) active layer. The structure of considered model consists of a $\mathrm{SiO}_{2} / \mathrm{Ta}_{2} \mathrm{O}_{5}$ optical $\mathrm{F}_{7}$ Fibonacci system, an organic active layer, and a thin silver layer on the top. Evaluation of the modal Purcell factor for the hybrid structure demonstrates significant enhancement of the spontaneous emission rate for an emitter, which is coupled with the Tamm plasmonlike states.
\end{abstract}

DOI: 10.12693/APhysPolA.136.653

PACS/topics: plasmonics, thin films, photonic quasicrystal, organics, Purcell effect

\section{Introduction}

Photonic quasicrystals (PQC) are a class of photonic structures, considered between ordered and fully disordered photonic systems $[1,2]$. PQCs are characterized by the long-range order and lack of periodicity. Such systems have also peculiar optical properties, e.g. the fractal density of photonic states and the light localization determined by this - even in $1 \mathrm{D}$ case [3-5]. The Tamm plasmons (TP) are electromagnetic states localized at the edge between the dielectric Bragg mirror and the metal. TP modes differ from conventional surface plasmon. They have some useful advantages like the existence inside the light cone (both in TE and TM polarizations) and lower absorption losses.

The use of metallic structures with photonic quasicrystal can lead to promising optical phenomena [6]. The topological properties of the TP-like state have been recently studied in a 1D photonic quasicrystal [7].

In this paper we theoretically study the hybrid Tamm plasmon - photonic quasicrystal cavity with an organic small molecule active layer. Light emission enhancement properties of this system and its general optical properties are analyzed.

\section{Structure description}

TP based cavity usually consists of a distributed Bragg reflector (DBR) and the metal layer, which surrounds the reflector. The DBR layer represents here the active layer. This is due to the fact that maximum of the electric field distribution of the Tamm plasmon mode is located

*corresponding author; e-mail: morzconst@gmail.com there. In our study, however, the DBR is replaced by the photonic quasicrystal system (optical Fibonacci lattice). Such a system represents a hybrid cavity with a different eigenmode structure. Scheme of the hybrid cavity is shown in Fig. 1. The cavity with optical Fibonacci lattice is constructed by the two base dielectric layers, namely A: $\mathrm{SiO}_{2}$ (silicon oxide) and $\mathrm{B}: \mathrm{Ta}_{2} \mathrm{O}_{5}$ (tantalum pentoxide), and by an organic active layer and the thin silver coating $(50 \mathrm{~nm})$. These materials are common ones in modern microcavity fabrication.

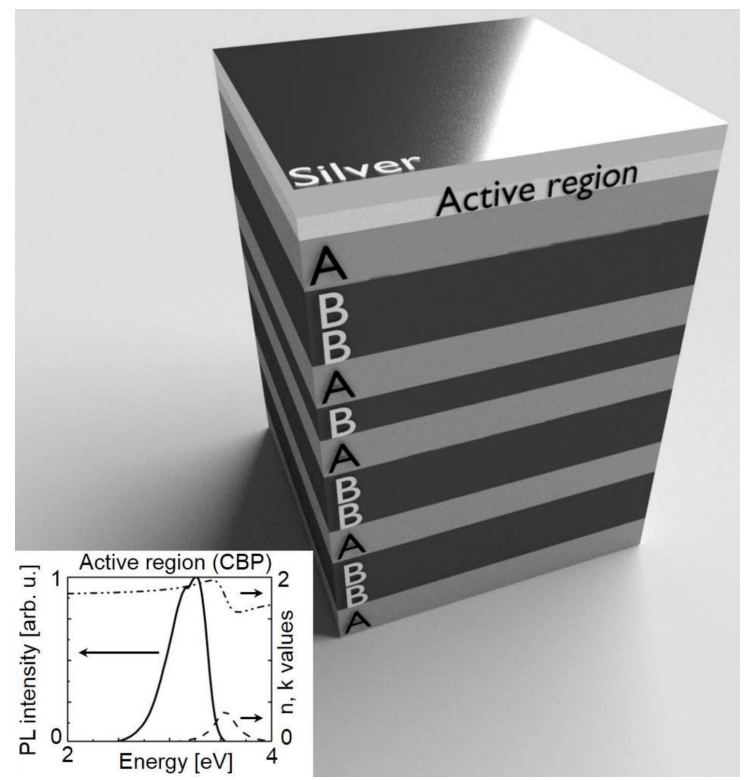

Fig. 1. Scheme of the hybrid Tamm plasmon - photonic quasicrystal structure with an organic active layer. The inset shows the optical properties of the CBP material (photoluminescence (PL) spectrum and refractive index distribution). 
The Fibonacci optical lattice is the simplest PQC. To construct the Fibonacci lattice sequence of order $F_{i+1}$ one uses the recurrent rule

$$
F_{i+1}=\left\{F_{i-1}, F_{i}\right\}
$$

with initial values: $F_{0}=\mathrm{A}\left(\mathrm{SiO}_{2}\right.$ layer for thickness $\left.d_{\mathrm{A}}=70 \mathrm{~nm}\right)$ and $F_{1}=\mathrm{B}\left(\mathrm{Ta}_{2} \mathrm{O}_{5}\right.$ layer for thickness $\left.d_{\mathrm{B}}=47.2 \mathrm{~nm}\right)$. The dielectric layer thickness corresponds to quarter-wavelength: $d_{\mathrm{A}, \mathrm{B}}=\lambda_{0} / 4 n_{\mathrm{A}, \mathrm{B}}$, where $n_{A, B}$ means refractive indexes of layers $\mathrm{A}\left(\mathrm{SiO}_{2}\right)$ and $\mathrm{B}\left(\mathrm{Ta}_{2} \mathrm{O}_{5}\right)$, respectively. In our studies, the $F_{7}$ order Fibonacci lattice was chosen (layer configuration BABABBABABBABBABABBA). Small organic molecule material 4,4'-bis(N-carbazolyl)-1,1'-biphenyl (CBP) [8] that emits in the violet and near UV spectral areas was chosen as the active layer. Optical properties of the CBP material are shown in inset of Fig. 1. The thickness of the CBP can be varied for mode spectral position tuning. Refractive indexes of the silicon oxide and tantalum pentoxide were taken from [9]. Optical parameters of the CBP came from experimental spectra fitting.

To investigate the optical properties of the hybrid TP - photonic quasicrystal - the cavity S-quantization formalism was used [10]. Assuming the TE polarization and the normal incidence of light, the reflectivity spectra were calculated. In case of TE polarization also the modal Purcell factor distribution was estimated.

\section{Results and discussion}

Reflectivity spectra of the conventional TP structure (Fig. 2a) and the hybrid cavity (Fig. 2b) with $30 \mathrm{~nm}$ thick CBP layer are calculated. The dashed lines indicate the reflectivity spectra of the DBR as well as of the $F_{7} \mathrm{Fi}-$ bonacci lattice. Reflectivity spectrum of the $F_{7}$ Fibonacci lattice is not fully symmetric compared to the Bragg frequency $(3 \mathrm{eV})$, which is related with material dispersion features of the $\mathrm{SiO}_{2}$ and $\mathrm{Ta}_{2} \mathrm{O}_{5}$. It can be seen that DBR has only one photonic bandgap (PBG) region, highlighted by the grey color. Due to the fractal density of photonic states of the optical Fibonacci lattices, the number of PGBs in the reflectivity spectrum will be higher than in the periodic case, and further, it will increase with the order of the lattice. PBG positions are centered at the Bragg frequency in each case. Thus, for each PBG region, one can find the frequency where the resonant condition is fulfilled and TP-like mode appeared. Comparing to the usual TP structure where the single TP mode appears, the hybrid $F_{7}$ cavity has five TP-like states. One state falls for each PBG. To estimate the quality factor for hybrid modes one can use the formula $Q=\omega_{0} / \Delta \omega$. The results of fitting of the reflectivity spectrum are the following: $Q \approx\{82,48,12,18,26\}$ (values are given from the lower to the higher energy of the mode). Note that the value for the conventional TP mode is $Q_{\mathrm{TP}} \approx 48$. Thus, in hybrid cavity case we have a set of modes with variety of $Q$-factors, however, the quality factor of the mode with the lowest energy is almost twice higher than the usual TP.
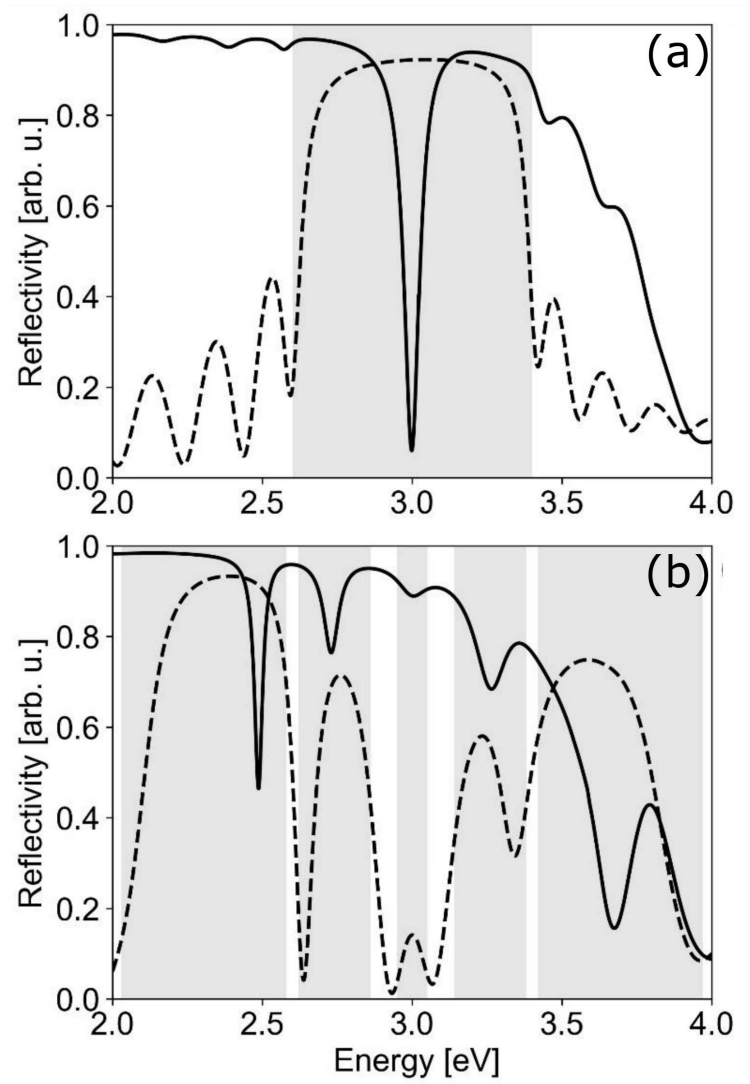

Fig. 2. (a) Reflectivity spectra of the DBR formed by $\mathrm{A}$ and $\mathrm{B}$ layers (dashed curve) and the conventional Tamm plasmon cavity formed from corresponding the DBR and metal (solid line). (b) Reflectivity spectra of the optical Fibonacci lattice of the $F_{7}$ order (dashed curve) and the hybrid $F_{7}$ Tamm plasmon cavity formed from corresponding Fibonacci lattice and metal (solid line). Grey area represents the PBG region for the DBR and the photonic quasicrystal systems.

Resonant energy of such states can be tuned by changing the thickness of the active layer, just like in the usual TP state. Figure 3 demonstrates the results of reflectivity spectra fitting - dependence of the spectral position on the thickness of the active CBP layer. Horizontal dashed lines represent the edges of the area of CBP emission and vertical dotted line shows the thickness corresponding to the $\lambda_{0} / 4$ value. It can be seen that for the $d_{\text {active }}<30 \mathrm{~nm}$ four low energy TP-like states have energies inside the CBP emission band.

To estimate the influence of the hybrid cavity environment on the organic molecule emission properties the analysis of the Purcell effect for the structure with $30 \mathrm{~nm}$ thick CBP layer was done. We have assumed that the considered system is operating in the weak coupling regime. Despite the existence of the strong exciton resonance in CBP at $3.52 \mathrm{eV}$ (the inset of Fig. 1) it turns out that dispersion of high- $Q$ TP-like states (two modes with lowest energies), that can efficiently interact with exciton, lies far from the exciton energy in this cavity design. 


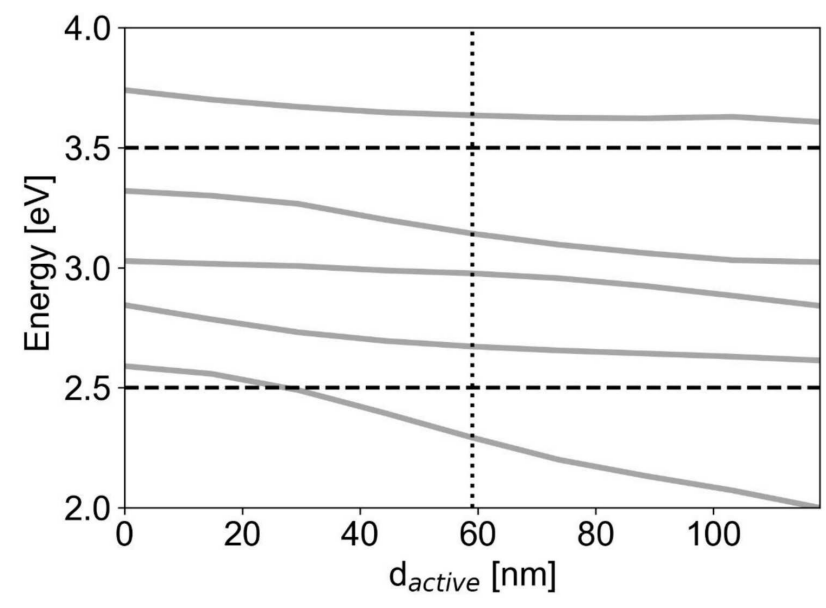

Fig. 3. Grey curves show the dependence of hybrid $F_{7}$ cavity's localized states spectral position on the thickness of the active layer. Horizontal dashed lines represent the edges of the area of the CBP emission. The vertical dotted line shows thickness corresponding to the $\lambda_{0} / 4$ value.

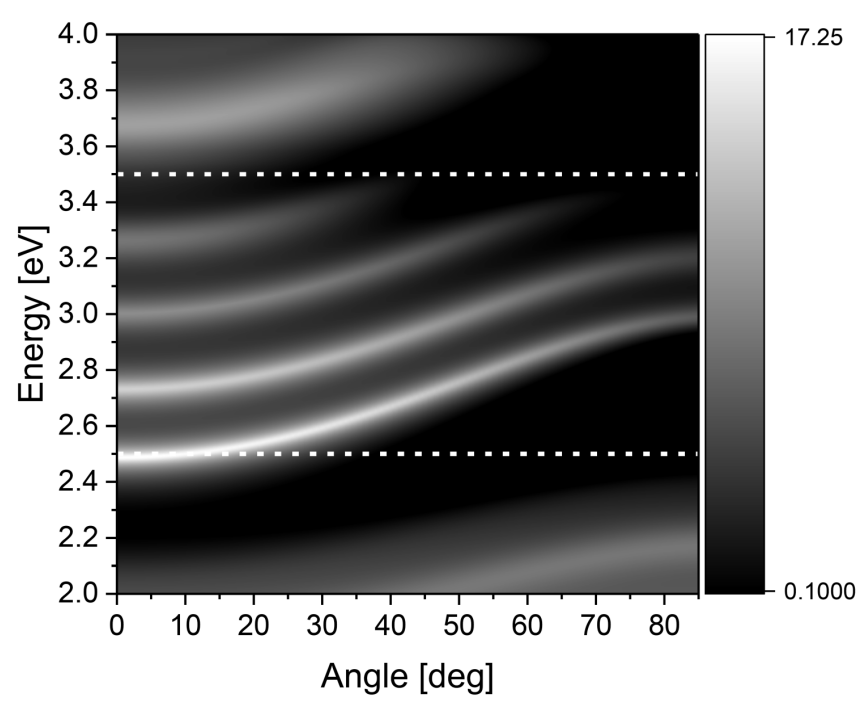

Fig. 4. Modal Purcell coefficient distribution for the $F_{7}$ hybrid TP cavity in case of the emitter placed in the active layer at $d=28 \mathrm{~nm}$ from the metal - active layer edge. Horizontal dashed lines represent the edges of the area of CBP emission.

By using $S$-quantization formalism [9] the modal Purcell factor dependence on the emission energy and the angle was calculated (Fig. 4). The emitter was placed at $28 \mathrm{~nm}$ from the edge between the cavity and metal. To evaluate Purcell coefficient in a well-known meaning, one needs to average the modal Purcell coefficient by all angles and polarizations. Spectral regions where significant enhancement of the dipole spontaneous emission rate takes place are in the $\mathrm{CBP}$ emission band. It can be seen that the dispersion of TP-like states is almost like the usual TP state. The maximum value of the modal
Purcell factor is up to 17 , corresponding to the lowest energy mode. Although, maximal modal Purcell factor for the hybrid structure does not strongly exceed the maximum value $(\approx 10)$ for the usual TP structure [11], this concept of structure can find different applications. Ability to control over the set of TP-like modes spectral position and $Q$-factor gives a few advantages over the usual single-mode TP. For instance, one of the modes can be used for effective optical pumping of the structure, while other ones for the emission enhancement.

Thus, noticeable enhancement of organic material spontaneous emission rate can be expected in the area corresponding to TP-like states in a wide spectral region. Furthermore, the increase of the optical Fibonacci lattice order can lead to the formation of the wide, almost continuous spectral area with many resonant states. Together with the emission band of given organic material the luminescent properties of organic material can be drastically modified, increasing the quantum efficiency.

\section{Conclusion}

The properties of the hybrid photonic quasicrystal the Tamm plasmon structure with an organic active layer have been theoretically analyzed. The dispersion and absorption of materials were taken into account in calculations. We have shown that the considered system supports the multiple Tamm plasmon-like hybrid states in the wide spectral region, corresponding to the CBP material emission band. Evaluation of the modal Purcell factor for the hybrid structure was carried out. Significant enhancement of the spontaneous emission rate for emitter coupled with Tamm plasmon-like states was demonstrated. The results show that hybrid TP-like states can be used for increase of the organic light-emitting material efficiency in the complex light emission systems.

\section{Acknowledgments}

This work has been supported by Russian Science Foundation (Project No. 16-12-10503).

\section{References}

[1] Z.V. Vardeny, A. Nahata, A. Agrawal, Nat. Photon. 7, 177 (2013).

[2] M.E. Zoorob, M.D.B. Charlton, G.J. Parker, J.J. Baumberg, M.C. Netti, Nature 404, 740 (2000).

[3] W. Gellermann, M. Kohmoto, B. Sutherland, P.C. Taylor, Phys. Rev. Lett. 72, 633 (1994).

[4] M.A. Kaliteevski, V.V. Nikolaev, R.A. Abram, S. Brand, Opt. Spectrosc. 91, 109 (2002).

[5] K.M. Morozov, K.A. Ivanov, A.R. Gubaydullin, M.A. Kaliteevski, Opt. Spectrosc. 122, 235-242 (2017).

[6] G.P. Swift, A.J. Gallant, N. Kaliteevskaya, M.A. Kaliteevski, S. Brand, D. Dai, A.J. Baragwanath, I. Iorsh, R.A. Abram, J.M. Chamberlain, Opt. Lett. 36, 1641 (2011). 
[7] M.K. Shukla, R. Das, Opt. Lett. 43, 362 (2018).

[8] V. Jankus, C. Winscom, A.P. Monkman, J. Chem. Phys. 130, 074501 (2009).

[9] L.V. Rodríguez-de Marcos, J.I. Larruquert, J.A. Méndez, J.A. Aznárez, Opt. Mater. Expr. 6, 3622 (2016).

[10] K.M. Morozov, E.I. Girshova, A.R. Gubaidullin, K.A. Ivanov, G. Pozina, M.A. Kaliteevski, J. Phys. Condens. Matter 30, 435304 (2018).

[11] A.R. Gubaydullin, C. Symonds, J. Bellessa, K.A. Ivanov, E.D. Kolykhalova, M.E. Sasin, A. Lemaitre, P. Senellart, G. Pozina, M.A. Kaliteevski, Sci. Rep. 7, 9014 (2017). 